\title{
On the mass transport in membraneless flow batteries with flow-by configuration
}

\author{
Kleber Marques Lisboa ${ }^{a, *}$, Renato Machado Cotta ${ }^{a, b}$ \\ a Laboratory of Nano- and Microfluidics and Microsystems, LabMEMS, Mechanical Engineering Dept. and Nanoengineering Dept., POLI E COPPE and Interdisciplinary Nucleus for \\ Social Development - NIDES/CT, Universidade Federal do Rio de Janeiro, Cidade Universitária, Cx. Postal 68503, Rio de Janeiro, RJ CEP 21945-970, Brazil \\ ${ }^{\mathrm{b}}$ Mechanical Engineering Dept., University College London, UCL, Roberts Building, Torrington Place, London WC1E 7JE, UK ${ }^{1}$
}

\section{A R T I C L E I N F O}

\section{Article history:}

Received 27 December 2017

Accepted 2 February 2018

Available online 20 February 2018

\section{Keywords:}

Membraneless redox flow batteries

Flow cells

Mass transfer

Integral transforms

Single domain formulation

Hybrid methods

\begin{abstract}
A B S T R A C T
Progress on the analysis of the mass transport phenomena in a membraneless redox flow battery with porous electrodes in flow-by configuration is here reported. A species transport model for a typical redox reactant interacting with a porous electrode is proposed. A hybrid numerical-analytical solution is obtained through the Generalized Integral Transform Technique (GITT), adopting a single domain formulation that includes both the porous and pure fluid regions. The influence of the Reynolds number and the thickness of the electrode on the parameters of interest is theoretically examined. The importance of considering the simultaneous development of flow and mass transport is analyzed, and the presence of the transversal convective flux of species proves to have a significant role on the generation of current inside the electrode. A scaling of the limiting current density with $\sim R e^{0.41}$ is demonstrated and some physical conclusions are drawn. Guidelines for the prevention of crossover are also offered, with increasing Reynolds number and decreasing relative thickness of the electrode having a positive effect, as far as avoiding the mixed potentials effects is concerned. The physical insights attained through the present analysis should add to the efforts in achieving membraneless redox flow batteries with performances comparable to membrane-based devices of similar size.
\end{abstract}

(c) 2018 Published by Elsevier Ltd.

\section{Introduction}

The challenge posed by climate change urged the rapid development of renewable energy sources, such as wind and solar. However, the intermittency associated with these power sources prevent the complete substitution of less environmental-friendly alternatives. In this context, the availability of efficient energy storage technologies is crucial to allow the reduction of emissions of certain pollutants from fossil fuels $[1,2]$. The redox flow battery (RFB) has been under development over the last three decades as an attractive energy storage option [3-5]. The main advantage of this concept is the decoupling of the energy and power densities, with the former being mainly determined by the size of the storage tanks, and the latter being mostly associated with the size of the battery itself. Moreover, deep charge-discharge cycles are possible without damaging the components, which contrasts with solidstate batteries [6].

\footnotetext{
* Corresponding author.

E-mail address: kleberlisboa@poli.ufrj.br (K.M. Lisboa).

1 Present address.
}

Besides the large-scale electrical energy storage, new usages for RFBs could arise from the miniaturization and decentralization of individual cells towards specific needs in different fields. The resulting diversification of applications could help RFBs attain lower costs, increasing the likelihood of its widespread usage [7]. One example where the use of RFBs would be a good fit is in electronics. The idea is to use flow battery technology for power delivery to the different electronic components. In fact, a fusing function of the well-studied heat removal $[8,9]$ and power delivery for data centers has been proposed [10] and demonstrated [11]. The basic concept is the use of the flux of electrolytes for both cooling and as energy source for individual electronic components, reducing the number of power connections and total wiring length. The perspective is the overall improvement of the energy efficiency and making more space available for logical connections in data centers [10].

Two strategies can be employed in the miniaturization of RFBs. The first consists in maintaining the constructive characteristics of large scale RFBs, solely reducing the size of its components, which has been successfully applied before $[11,12]$. The second one takes advantage of the laminar flow regime in channels with small crosssections to limit the mixture of electrolytes at the interface 


\begin{tabular}{|c|c|c|c|}
\hline \multicolumn{4}{|c|}{ Nomenclature } \\
\hline$c$ & concentration & & \\
\hline$u$ & longitudinal velocity component & \multicolumn{2}{|c|}{ Greek letters } \\
\hline$v$ & transversal velocity component & $\sigma$ & $=d_{f} / h$ \\
\hline$\nabla$ & nabla operator: $\left(\frac{\partial}{\partial x}, \frac{\partial}{\partial y}, \frac{\partial}{\partial z}\right)$ & $\varepsilon$ & porosity of the electrode \\
\hline$h$ & half of the channel height & $\beta, v$ & eigenvalues corresponding to eigenfunctions $\psi$ and $\xi$, \\
\hline$h_{p}$ & thickness of the porous layer & & respectively \\
\hline$L^{*}$ & channel length & $\psi$ & eigenfunction for the concentration \\
\hline $\operatorname{Re}$ & Reynolds number & $\xi$ & auxiliary eigenfunction \\
\hline$D a$ & Darcy number & $\delta$ & Kronecker delta \\
\hline $\mathrm{Pe}$ & Péclét number & & \\
\hline Sc & Schmidt number & \multicolumn{2}{|c|}{ Subscripts and superscripts } \\
\hline$u_{f}$ & fully developed velocity profile & $i, j, k$ & order of eigenquantities for the concentration and \\
\hline$c_{\text {in }}$ & entry concentration profile & & velocity vector \\
\hline$x_{0}$ & dimensionless channel length & & order of auxiliary eigenquantities \\
\hline$w_{p}$ & relative thickness of the porous electrode & $\sim$ & normalized eigenfunction \\
\hline$d_{f}$ & diameter of the pore or the fiber of the electrode & & dimensional quantity \\
\hline$a$ & specific area of the electrode & & transpose \\
\hline$k_{m}$ & mass transfer coefficient & & bulk quantity \\
\hline M & eigenfunction truncation order & & \\
\hline
\end{tabular}

between them, enabling the removal of the membrane in RFBs and fuel cells [13-15]. The so-called membraneless co-laminar flow cells are simpler and cheaper to manufacture in comparison with membrane-based ones with similar size, mainly due to the absence of the costly ion-selective membrane [16]. However, membraneless RFBs suffer from low Reynolds diffusion-limited mass transport, severely limiting the overall performance of these devices. Several strategies have been reported for increasing the mass transport performance of membraneless flow cells, from the use of porous carbon electrodes [15,17-19], to the employment of chaotic mixers [20-22]. Disrupting the diffusion-limited transport has the critical side-effect of doing the same to the interface between the two electrolytes, making crossover and mixed potential phenomena more likely. Addressing the two aforementioned issues together strongly benefits the overall performance of membraneless flow cells, leading to a maximum power density of 0.925 $\mathrm{W} / \mathrm{cm}^{2}$ recently reported in the literature [23]. This power density is on the same order magnitude to the ones attained with membrane-based microfluidic RFBs [11,12]. Nevertheless, basic understanding of the transport phenomena in membraneless colaminar flow cells is still lacking, especially for the ones employing porous carbon electrodes.

Experimental assessment of the performance of RFBs with different configurations and sizes can be quite cumbersome and expensive. For this reason, a few numerical simulation efforts have been made to simulate flow cells in both membrane-based [24,25] and membraneless [26,27] systems. The availability of versatile discrete numerical methods such as finite elements and finite volumes has enabled the evaluation of the performance of RFBs. On the other hand, analytical methods provide valuable physical insights, low cost solutions to any prescribed accuracy, and benchmark results for the verification of numerical codes. The value of analytically-based methods has been recognized before in the context of RFBs, with a boundary layer analysis of a membraneless hydrogen-bromine flow battery [28,29]. However, analytical methods are usually restricted to linear problems in regular geometries, which severely limits their applicability. With the intent of extending the use of analytical methods beyond these limitations, the socalled hybrid analytical-numerical methods were proposed. Amongst such hybrid methods, the Generalized Integral Transform Technique (GITT) [30-34] is here highlighted. The GITT was shown to be a fairly general technique, being demonstrated in the solution of various classes of diffusion and convection-diffusion problems, such as with moving boundaries, non-linear source terms, heterogeneous media, complex geometries, etc. The main advantages of this method are the automatic error control and mild increase in computational cost with the addition of independent variables. A complete description and broad review of the technique can be found in some compilations published over the years [30-34].

Within the GITT framework, a new strategy for treating problems in heterogeneous media with complex geometries, known as the single domain formulation, has been under development [35-40] and was recently applied to the solution of heterogeneous channel flow governed by the Navier-Stokes equations [41]. Instead of treating the different domains with separate partial differential models coupled through their common boundaries, the single domain formulation proposes a single set of equations in which the coupling between the different media is accomplished through abrupt variations of the physical properties and source terms. The reduced complexity that follows offers far-reaching possibilities for the application of the GITT in classes of problems once mostly dominated by purely numerical approaches.

The present work advances the theoretical analysis and physical understanding of the mass transport phenomena within the scope of membraneless redox flow batteries. A simple case of a cell with flow-by configuration using porous electrodes is analyzed. Both analytical and hybrids solutions to the involved partial differential models for different flow conditions are presented, and critical comparisons are offered. The role of the Reynolds number and the thickness of the electrode on the prevention of crossover is analyzed. The scaling of the dimensionless limiting current density with the Reynolds number and the physical interpretation of this quantity are assessed. Additionally, the dependence of the limiting current with the length of the electrode is examined and some design recommendations are offered.

\section{Formulation and solution methodology}

\subsection{Fluid flow model}

Fig. 1 depicts the physical situation involving the hydrodynamic and mass transport simultaneous development along a parallel plate channel partially filled with porous media. A Cartesian coor- 


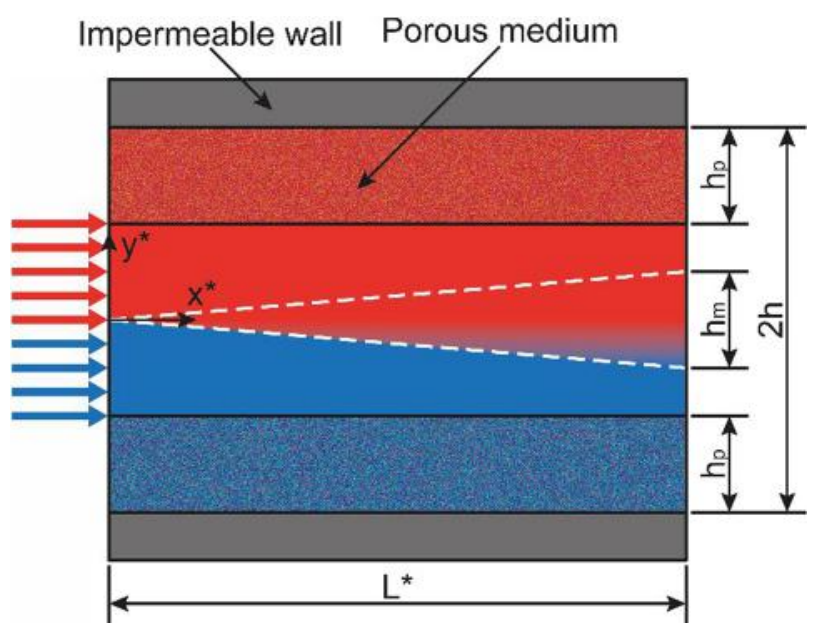

Fig. 1. Schematic representation of the mass transport problem in a parallel plate channel partially filled with porous media. Mixture zone illustrated by white dashed line.

dinate system is conveniently positioned on the centerline of the channel. The flow direction and its uniformity are indicated by the arrows positioned at the entry of the channel. The red color represents the zone with significant electrolyte presence, with the inverse being true for the blue colored zone, in which the presence of electrolyte becomes negligible. The region delimited by the white dashed lines developing from the origin of the Cartesian coordinate system represents the zone affected by the diffusion of the electrolyte to the oppose side, in a phenomenon called crossover. The thickness of this zone at the outlet of the channel $\left(h_{m}\right)$ is particularly important and is indicated in Fig. 1 together with other dimensions of interest. The presence of impermeable walls and the location of the porous media positioned in a transversally symmetrical manner are also highlighted.

The fluid flow is modeled in two ways, depending on the medium under consideration. For the free fluid, it is used standard steady-state continuity and Navier-Stokes equations for incompressible flow with constant physical properties. Within the porous electrodes, the Darcy equation with the Brinkman correction was deemed the appropriate model. Nevertheless, the single domain formulation allows for the unification of these two fluid flow models, differentiating them through spatially variable physical properties. The resulting governing equations for the fluid flow, in dimensionless form, are shown below:

$\nabla \cdot \boldsymbol{u}=0$

$\rho(\boldsymbol{u} \cdot \nabla) \boldsymbol{u}=-\nabla p+\frac{4}{\operatorname{Re}} \nabla \cdot[\mu \nabla \boldsymbol{u}]-\frac{4 \gamma}{\operatorname{ReDa}} \boldsymbol{u}$

where $\boldsymbol{u}$ is the dimensionless velocity vector, $\nabla$ is the dimensionless nabla operator, $\rho$ is the dimensionless density, $p$ is the dimensionless pressure field, $\mu$ is the dimensionless dynamic viscosity, $R e$ is the Reynolds number based on the hydraulic diameter, and $D a$ is the Darcy number.

The dimensionless quantities can be obtained from the respective dimensional quantities through the following expressions:

$\boldsymbol{u}=\frac{\boldsymbol{u}^{*}}{u_{0}} ; \nabla=h \nabla^{*} ; \rho=\frac{\rho^{*}}{\rho_{0}} ; p=\frac{p^{*}}{\rho_{0} u_{0}^{2}} ;$

$\mu=\frac{\mu^{*}}{\mu_{0}} ; \quad R e=\frac{4 \rho_{0} u_{0} h}{\mu_{0}} ; D a=\frac{\kappa}{h^{2}}$

where $\boldsymbol{u}^{*}$ is the velocity vector, $u_{0}$ is the uniform entry longitudinal velocity component, $\nabla^{*}$ is the nabla operator, $\rho^{*}$ is the density, $\rho_{0}$ is the fluid density, $p^{*}$ is the pressure field, $\mu^{*}$ is the viscosity, $\mu_{0}$ is the fluid viscosity, and $\kappa$ is the permeability of the porous medium.

Let $V_{f}$ and $V_{p}$ be the control volumes corresponding to the free fluid and the porous media, respectively. The spatially variable physical properties are defined as follows:

$\rho= \begin{cases}1, & \text { in } V_{f} \\ 0, & \text { in } V_{p}\end{cases}$

$\mu= \begin{cases}1, & \text { in } V_{f} \\ 1 / \varepsilon, & \text { in } V_{p}\end{cases}$

$\gamma= \begin{cases}0, & \text { in } V_{f} \\ 1, & \text { in } V_{p}\end{cases}$

where $\varepsilon$ is the porosity of the porous medium.

In the entry of the free fluid region, a uniform velocity profile is imposed. In the remaining entry section and at the wall contour a null velocity vector boundary condition is used. At the outlet, a fully developed velocity profile, $u_{f}$, is assumed.

A new methodology for obtaining the solution of the fluid flow through integral transforms in this physical situation was recently reported [41]. In that work, the velocity vector was decomposed on a base flow and on an infinite number of fundamental vortices, which translates into the following expression:

$\boldsymbol{u}(x, y)=\boldsymbol{u}_{f}(y)+\sum_{i=1}^{\infty} \nabla \times\left[\bar{\phi}_{i}(x) \boldsymbol{\Phi}_{i}(y)\right]$

where

$\begin{aligned} \boldsymbol{u}_{f}(y) & =\left[\begin{array}{lll}u_{f}(y) & 0 & 0\end{array}\right]^{T} \\ \boldsymbol{\Phi}_{i}(y) & =\left[\begin{array}{lll}0 & 0 & \tilde{\varphi}_{i}(y)\end{array}\right]^{T}\end{aligned}$

For a complete description of the solution methodology and of the algorithm developed for the solution of the fluid flow under the conditions herein described, we refer the reader to Ref. [41].

\subsection{Species transport model}

The model chosen to represent the physical situation of Fig. 1 is the steady-state two-dimensional convection-diffusion of diluted species with constant molecular diffusivities. A source term is included to represent the electrochemical reactions within the porous electrode. Two situations are considered here. The first one is that the flow is fully developed since the entry, and only the depletion boundary layers are developing. In the second situation, as depicted in Fig. 1, simultaneous development is explored with velocity vector field being determined with the methodology of Ref. [41]. To avoid the complications associated with the solution of different partial differential models for each sub-domain of a heterogeneous medium coupled by their common boundaries, the single domain formulation is employed also for the mass transport model.

Regarding the boundary conditions, at the entry, a concentration profile varying with the transversal coordinate is imposed. In the zones indicated as walls at the top and the bottom of Fig. 1, impermeable wall boundary conditions are applied. Considering the invariance of the concentration profile with the longitudinal coordinate under fully developed conditions, a boundary condition with null concentration gradient is proposed at a longitudinal position tending to infinity.

The above described model assumes the following mathematical form for the Cartesian coordinate system of Fig. 1:

$u(x, y) \frac{\partial c}{\partial x}+v(x, y) \frac{\partial c}{\partial y}=\frac{4}{P e}\left\{D(y) \frac{\partial^{2} c}{\partial x^{2}}+\frac{\partial}{\partial y}\left[D(y) \frac{\partial c}{\partial y}\right]\right\}+S(x, y)$ 
with inlet and outlet boundary conditions given by:

$c(0, y)=c_{\text {in }}(y) ;\left.\quad \frac{\partial c}{\partial x}\right|_{x \rightarrow \infty}=0$

and remaining boundary conditions given by:

$\left.\frac{\partial c}{\partial y}\right|_{y=-1}=0 ;\left.\quad \frac{\partial c}{\partial y}\right|_{y=1}=0$

where $u$ is the dimensionless longitudinal velocity component, $v$ is the dimensionless transversal velocity component, $c$ is the dimensionless concentration, $D$ is the dimensionless diffusivity, $P e$ is the Péclét number, and $S$ is the source term related to the electrochemical reactions.

The dimensional quantities can be obtained from their dimensionless counterparts in the following way:

$x=\frac{x^{*}}{h} ; \quad y=\frac{y^{*}}{h}$

$u=\frac{u}{u_{0}} ; \quad v=\frac{v}{u_{0}} ; \quad c=\frac{c^{*}}{c_{0}}$

$D=\frac{D^{*}}{D_{0}} ; \quad P e=\operatorname{ReSc}=\frac{4 u_{0} h}{D_{0}}$

where $c_{0}$ is the uniform electrolyte concentration imposed in the region indicated with red arrows in Fig. $1, c^{*}$ is the dimensional concentration, $D_{0}$ is the electrolyte diffusivity, and $S c$ is the Schmidt number $\left(\mu_{0} /\left(\rho_{0} D_{0}\right)\right)$.

Following the single domain formulation strategy, applying the Bruggeman effective diffusivity model [42], and defining the entry concentration profile in accordance with Fig. 1, we then have,

$D(y)= \begin{cases}1, & -1+w_{p} \leqslant y \leqslant 1-w_{p} \\ \varepsilon^{1.5}, & y<-1+w_{p} \text { or } y>1-w_{p}\end{cases}$

$c_{\text {in }}(y)= \begin{cases}1, & 0 \leqslant y \leqslant 1-w_{p} \\ 0, & y<0 \text { or } y>1-w_{p}\end{cases}$

where $w_{p}$ is the ratio between the thickness of the electrode $\left(h_{p}\right.$ in Fig. 1) and half of the channel width ( $h$ in Fig. 1), henceforth referred as relative electrode thickness.

In this work, the velocity components shall be defined in two different ways. In the first formulation, under fully developed flow conditions, the velocity vector must be equal to $\boldsymbol{u}_{f}$, as defined in Eq. (7.b). The result is shown below:

$u(x, y)=u_{f}(y)$

$v(x, y)=0$

For the case involving simultaneous development, the expansion of the curl operator at Eq. (7.a) with respect to the Cartesian coordinate system of Fig. 1 is carried out, resulting in:

$u(x, y)=u_{f}(y)+\sum_{i=1}^{\infty} \bar{\phi}_{i}(x) \frac{d \tilde{\varphi}_{i}}{d y}$

$v(x, y)=-\sum_{i=1}^{\infty} \frac{d \bar{\phi}_{i}}{d x} \tilde{\varphi}_{i}(y)$

The source term is built upon the assumption that the electrolyte consumption occurs at a rate that is proportional to the difference between the concentrations of the bulk of the fluid and at the reactive surfaces. To analyze the performance from a mass transport perspective only and to drop the need to introduce complicated models for the electrochemical reaction kinetics, the lim- iting current conditions are here employed. In other words, it is assumed that the timescale for the electrochemical reactions is a lot smaller than the timescale for the transport of electrolytes to the reactive surfaces, allowing the imposition of a null concentration at the active areas of the porous electrodes [28]. Therefore, the source term can be written as [24,25,27]:

$S(x, y)=-a h k_{m} \zeta(y) c(x, y)$

with,

$\zeta(y)= \begin{cases}1, & \text { in } 1-w_{p} \leqslant y \leqslant 1 \\ 0, & \text { in }-1<y<1-w_{p}\end{cases}$

where $a$ is the specific area of the active surfaces of the porous electrodes and $k_{m}$ is the dimensionless mass transfer coefficient.

Assuming the mass transport within the porous electrode occurs mainly through diffusion, neglecting the convective effects over the fibers or pores of the electrode, the mass transfer coefficient can be determined using the following expression [24,25]:

$k_{m}^{*}=\frac{D_{0}}{d_{f}}$

where $k_{m}^{*}$ is the dimensional mass transfer coefficient and $d_{f}$ is the diameter of the fibers or the pores of the electrode. Defining,

$k_{m}=\frac{k_{m}^{*}}{u_{0}}$

we then have,

$k_{m}=\frac{4}{\sigma P e}$

where $\sigma$ is the ratio between the diameter of the fiber or the pore of the electrode, $d_{f}$, and half the channel height, $h$.

One last simplification is possible realizing that the Péclét number, for the conditions to be tested in this work (see Section 2.7), is fairly high, mainly due to the Schmidt numbers on the order of $\sim 100$. This fact allows the neglection of the longitudinal diffusion effects, eliminating the need to impose the boundary condition, as previously discussed, in terms of the invariance of the concentration profile as the longitudinal coordinate tends to infinity. Hence, the simplified partial differential model becomes:

$u \frac{\partial c}{\partial x}+v \frac{\partial c}{\partial y}=\frac{4}{P e}\left\{\frac{\partial}{\partial y}\left[D(y) \frac{\partial c}{\partial y}\right]-\frac{a h}{\sigma} \zeta(y) c(x, y)\right\}$

with entry condition given by:

$c(0, y)=c_{\text {in }}(y)$

and the remaining boundary conditions given by:

$\left.\frac{\partial c}{\partial y}\right|_{y=-1}=0 ;\left.\quad \frac{\partial c}{\partial y}\right|_{y=1}=0$

\subsection{Eigenvalue problem}

Substituting the fully developed velocity components of Eqs. (11.a,b) into Eq. (17.a) and applying the method of separation of variables, the following Sturm-Liouville eigenvalue problem is obtained [30-34]:

$\frac{d}{d y}\left[D(y) \frac{d \psi_{i}}{d y}\right]+\left[\beta_{i}^{2} u_{f}(y)-\frac{a h}{\sigma} \zeta(y)\right] \psi_{i}(y)=0$

with boundary conditions and normalization given by:

$\left.\frac{d \psi_{i}}{d y}\right|_{y=-1}=0 ;\left.\quad \frac{d \psi_{i}}{d y}\right|_{y=1}=0$ 
$\tilde{\psi}_{i}(y)=\frac{\psi_{i}(y)}{\sqrt{N_{\psi, i}}} ; \quad N_{\psi, i}=\int_{-1}^{1} u_{f}(y) \psi_{i}(y)^{2} d y$

The solution of the eigenvalue problem shown above bears the following orthogonality property:

$\int_{-1}^{1} u_{f}(y) \tilde{\psi}_{i}(y) \tilde{\psi}_{j}(y) d y=\delta_{i j}$

With the help of the orthogonality property, a transforminverse pair can be formed, as follows:

$\bar{c}_{i}(x)=\int_{-1}^{1} u_{f}(y) \tilde{\psi}_{i}(y) c(x, y) d y$

$c(x, y)=\sum_{i=1}^{\infty} \bar{c}_{i}(x) \tilde{\psi}_{i}(y)$

The impossibility to obtain analytical solutions for Eqs. (18.a-e) brings the need of a hybrid solution methodology to the eigenvalue problem. The GITT itself is chosen to perform the solution of the eigenvalue problem here proposed. Before proceeding with the integral transformation of Eq. (18.a), a simpler eigenvalue problem must be proposed, whose solution shall serve as a basis for expanding the eigenfunction $\psi_{i}$. The simplest amongst the Sturm-Liouville eigenvalue problems is selected, as shown below:

$\frac{d^{2} \xi_{m}}{d y^{2}}+v_{m}^{2} \xi_{m}(y)=0$

with boundary conditions and normalization given by:

$\left.\frac{d \xi_{m}}{d y}\right|_{y=-1}=0 ;\left.\quad \frac{d \xi_{m}}{d y}\right|_{y=1}=0$

$\tilde{\xi}_{m}(y)=\frac{\xi_{m}(y)}{\sqrt{N_{\xi, m}}} ; \quad N_{\xi, m}=\int_{-1}^{1} \xi_{m}^{2}(y) d y$

The eigenvalue problem of Eqs. (21.a-c) admits analytical solution in the form,

$\tilde{\xi}_{m}(y)=\left\{\begin{array}{l}1 / \sqrt{2}, \text { for } m=1 \\ \cos \left((m-1) \pi \frac{y+1}{2}\right), \text { for } m>1\end{array}\right.$

The eigenfunction defined by the expression in Eq. (22) has the following orthogonality property:

$\int_{-1}^{1} \tilde{\xi}_{m}(y) \tilde{\xi}_{n}(y) d y=\delta_{m n}$

Following the GITT formalism, a transform-inverse pair is then formed for the eigenfunction $\psi_{i}$ :

$\bar{\psi}_{i, m}=\int_{-1}^{1} \tilde{\xi}_{m}(y) \psi_{i}(y) d y$

$\psi_{i}(y)=\sum_{m=1}^{\infty} \bar{\psi}_{i, m} \tilde{\xi}_{m}(y)$

Operating Eq. (18.a) with $\int_{-1}^{1} \tilde{\xi}_{m}(y)_{--} d y$, substituting the inverse formula of Eq. (24.b), and using integration by parts to avoid derivatives involving discontinuous functions, the algebraic eigenvalue problem below results:

$\left(\boldsymbol{P}+\beta_{i}^{2} \mathbf{Q}\right) \bar{\psi}_{i}=0$

$\bar{\psi}_{i}=\left\{\bar{\psi}_{i, m}\right\} ; \boldsymbol{P}=\left\{P_{m n}\right\} ; \boldsymbol{Q}=\left\{Q_{m n}\right\}$

with integral coefficients given by,
$P_{m n}=-\int_{-1}^{1}\left[D(y) \frac{d \tilde{\xi}_{m}}{d y} \frac{d \tilde{\xi}_{n}}{d y}+\frac{a h}{\sigma} \zeta(y) \tilde{\xi}_{m}(y) \tilde{\xi}_{n}(y)\right] d y$

$Q_{m n}=\int_{-1}^{1} u_{f}(y) \tilde{\xi}_{m}(y) \tilde{\xi}_{n}(y) d y$

The numerical values for the integral coefficients of Eqs. (25.e,f) are calculated using analytically obtained formulae employing mixed symbolic-numerical routines built-in Mathematica v. 10.4 platform [43]. To obtain the solution to the algebraic eigenvalue problem of Eqs. (25.a-f), truncation to a finite order $M$ is needed. Robust numerical routines available in the software Mathematica v. 10.4 [43] are then used to carry out the calculations of the eigenvalues and the corresponding eigenvectors. Finally, the eigenvectors are fed into the inverse formula of Eq. (24.b) to obtain the eigenfunctions $\psi_{i}$.

\subsection{Transformed problem}

Applying the operator $\int_{-1}^{1} \tilde{\psi}_{i}(y)_{--} d y$ to Eq. (17.a), rearranging, employing integration by parts when necessary, using Eq. (18.a), and, finally, substituting the inverse formula of Eq. (20.b) into the results, the transformed system of ordinary differential equations below is obtained:

$\sum_{j=1}^{\infty}\left\{A_{i j}(x) \frac{d \bar{c}_{j}}{d x}+B_{i j}(x) \bar{c}_{j}(x)\right\}+\frac{4 \beta_{i}^{2}}{P e} \bar{c}_{i}(x)=0$

with integral coefficients given by:

$A_{i j}(x)=\int_{-1}^{1} u(x, y) \tilde{\psi}_{i}(y) \tilde{\psi}_{j}(y) d y$

$B_{i j}(x)=\int_{-1}^{1} v(x, y) \tilde{\psi}_{i}(y) \frac{d \tilde{\psi}_{j}}{d y} d y$

The entry condition must be transformed in a similar way. Operating Eq. (17.b) with $\int_{-1}^{1} u_{f}(y) \tilde{\psi}_{i}(y)_{-} d y$, it is then obtained:

$\bar{c}_{i}(0)=\bar{c}_{i n, i}=\int_{-1}^{1} u_{f}(y) \tilde{\psi}_{i}(y) c_{i n}(y) d y$

For the analysis in this work, two flow conditions are considered. In the first, the flow is assumed to be fully developed and the velocity components are given by Eqs. (11.a,b). Substituting both equations into Eqs. (26.b,c) and employing the orthogonality property of Eq. (19), the following decoupled transformed system results:

$\frac{d \bar{c}_{i}}{d x}+\frac{4 \beta_{i}^{2}}{P e} \bar{c}_{i}(x)=0$

The transformed system of Eq. (28), together with the entry condition of Eq. (27), lead to a simple analytical solution in the form:

$\bar{c}_{i}(x)=\bar{c}_{i n, i} \exp \left(-\frac{4 \beta_{i}^{2}}{P e} x\right)$

In the case with simultaneous development, the velocity components are given by Eqs. (12.a,b). Substituting those expressions into Eqs. (26.b,c) and rearranging, the transformed problem becomes:

$\sum_{j=1}^{\infty}\left\{A_{i j}(x) \frac{d \bar{c}_{j}}{d x}+B_{i j}(x) \bar{c}_{j}(x)\right\}+\frac{4 \beta_{i}^{2}}{P e} \bar{c}_{i}(x)=0$

with entry condition given by: 
$\bar{c}_{i}(0)=\bar{c}_{i n, i}$

and integral coefficients given by:

$$
\begin{aligned}
& A_{i j}(x)=\delta_{i j}+\sum_{k=1}^{\infty}\left\{\int_{-1}^{1} \tilde{\psi}_{i}(y) \tilde{\psi}_{j}(y) \frac{d \tilde{\varphi}_{k}}{d y} d y\right\} \bar{\phi}_{k}(x) \\
& B_{i j}(x)=-\sum_{k=1}^{\infty}\left\{\int_{-1}^{1} \tilde{\psi}_{i}(y) \frac{d \tilde{\psi}_{j}}{d y} \tilde{\varphi}_{k}(y) d y\right\} \frac{d \bar{\phi}_{k}}{d x}
\end{aligned}
$$

\subsection{Bulk concentration and limiting current density}

With the consumption of electrolytes at the porous electrode, a quantity capable of measuring the amount of reactants still available for the electrochemical reactions would be convenient. The bulk concentration can have this role by establishing an average weighted by the longitudinal velocity component for each position $x$, allowing a direct evaluation of the portion of electrolytes fed into the cell that gets carried along with the flow. Mathematically,

$c_{b}(x)=\frac{\int_{-1}^{1} u(x, y) c(x, y) d y}{\int_{-1}^{1} u(x, y) d y}$

Invoking the mass conservation principle, the following must be true:

$\int_{-1}^{1} u(x, y) d y=2\left(1-w_{p}\right)$

The general expression for the bulk concentration then becomes:

$c_{b}(x)=\frac{1}{2\left(1-w_{p}\right)} \int_{-1}^{1} u(x, y) c(x, y) d y$

Due to the dependency on the longitudinal component of the velocity vector, the bulk concentration is also subject to the two hydrodynamic development cases studied in this work. Applying the fully developed velocity profile from Eq. (11.a), substituting the inverse formula of Eq. (20.b), and employing the solution to the transformed problem for this case (Eq. (29)), we then have,

$c_{b}(x)=\frac{1}{2\left(1-w_{p}\right)} \sum_{i=1}^{\infty} \bar{c}_{i n, i} g_{i} \exp \left(-\frac{4 \beta_{i}^{2}}{P e} x\right)$

with integral coefficient given by:

$g_{i}=\int_{-1}^{1} u_{f}(y) \tilde{\psi}_{i}(y) d y$

For the more general case, involving the simultaneous development of the velocity and concentration profiles, the longitudinal velocity component of Eq. (12.a) is substituted into Eq. (33), followed by the use of the inverse formula of Eq. (20.b). The result is shown below:

$c_{b}(x)=\frac{1}{2\left(1-w_{p}\right)}\left\{\sum_{i=1}^{\infty} g_{i} \bar{c}_{i}(x)+\sum_{k=1}^{\infty} \sum_{i=1}^{\infty} q_{i k} \bar{\phi}_{k}(x) \bar{c}_{i}(x)\right\}$

with integral coefficients given by:

$g_{i}=\int_{-1}^{1} u_{f}(y) \tilde{\psi}_{i}(y) d y ; \quad q_{i k}=\int_{-1}^{1} \tilde{\psi}_{i} \frac{d \tilde{\varphi}_{k}}{d y} d y$

The instantaneous consumption of the electrolytes upon contact with the reactive surfaces of the porous electrodes has been assumed in the modeling process. Therefore, the electrical current obtained from the results of this work is the limiting current itself. Amongst the modeled quantities, the one that bears relation to the reaction rates is the source term $S$. The relation between the source term and the limiting current is determined through the integration of $S$ along a volume of variable length, $x^{\prime}$, and occupying the whole cross-section of the electrode at the top of Fig. 1. The limiting current density is then obtained with the normalization of the result of the integration by the footprint of the cell (the product of the width in $z$-direction and the variable length of the electrode). The expression for the limiting current density is the following:

$i_{\text {lim }}^{*}\left(x^{\prime}\right)=\frac{n F u_{0} c_{0}}{x^{\prime}} \int_{0}^{x^{\prime}} \int_{1-w_{p}}^{1} \frac{4}{P e} \frac{a h}{\sigma} c(x, y) d y d x$

where $i_{\text {lim }}^{*}$ is the dimensional limiting current density, $n$ is the number of electrons involved in the electrochemical reactions, $F$ is Faraday's constant $(96,485 \mathrm{C} / \mathrm{mol})$, and $x^{\prime}$ is the length of the electrode considered in the calculation of the limiting current.

In order to assure the generality of the analysis, a definition for a dimensionless current density is needed. Defining,

$i_{\text {lim }}=\frac{i_{\text {lim }}^{*}}{n F c_{0} u_{0}}$

one then has,

$i_{\text {lim }}\left(x^{\prime}\right)=\frac{1}{x^{\prime}} \int_{0}^{x^{\prime}} \int_{1-w_{p}}^{1} \frac{4}{P e} \frac{a h}{\sigma} c(x, y) d y d x$

where $i_{\text {lim }}$ is the dimensionless limiting current density.

Employing the operator $\frac{1}{x^{\prime}} \int_{0}^{x^{\prime}} \int_{-1}^{1}-d y d x$ on Eq. (17.a), knowing the transversal velocity component is zero at the walls, using the boundary conditions of Eq. (17.c,d), the definition of Eq. (13.b), and the expression for the bulk concentration of Eq. (33), it results,

$\frac{1}{x^{\prime}} \int_{0}^{x^{\prime}} \int_{1-w_{p}}^{1} \frac{4}{P e} \frac{a h}{\sigma} c(x, y) d y d x=\frac{2\left(1-w_{p}\right)}{x^{\prime}}\left[c_{b}(0)-c_{b}\left(x^{\prime}\right)\right]$

Therefore, the limiting current density can be written as:

$i_{\text {lim }}\left(x^{\prime}\right)=\frac{2\left(1-w_{p}\right)}{x^{\prime}}\left[c_{b}(0)-c_{b}\left(x^{\prime}\right)\right]$

The relation between the bulk concentration and the dimensionless limiting current density of Eq. (40) demonstrates that the latter is a direct measure of the average depletion of the electrolytes as the flow advances along the parallel plate channel.

\subsection{Solution procedure}

The velocity components for both the fully developed and the simultaneous development cases, as mentioned before, are obtained with the GITT methodology described elsewhere [41]. Due to the independence of the flow equations from the results of the mass transport analysis, the velocity vector field is obtained in a prior step to the solution of the model for the species transport.

Before proceeding with the solution of the transformed problem, numerical values for the integral coefficients at the entry condition and the system of ordinary differential equations itself must be available. Substituting the inverse formula for the eigenfunction of Eq. (24.b) into the integral coefficients of Eqs. (27) and (30.c,d), and rearranging the summations, integrals involving the simpler eigenfunction $\tilde{\xi}_{m}$ arise. The integrals in terms of the simpler eigenfunctions can be easily evaluated from general formulae analytically obtained with symbolic computation routines present in the software Mathematica v. 10.4 [43].

The transformed systems for both the solution of the eigenvalue problem and the species transport model involve an infinite number of equations and dependent variables. Due to the impossibility of solving such infinite systems, both sets of equations must be truncated. The final truncation order of each one is directly gov- 
erned by how many terms are included in the summations of Eqs. (20.b) and (24.b). In this work, the number of terms used in the eigenfunction expansions are $N$ and $M$ for the concentration and the eigenfunction $\psi_{i}$, respectively. These two truncation orders are the only parameters to be monitored as far as convergence is concerned.

The system of ordinary differential equations, whose solution must be used to obtain the concentration from the eigenfunction expansion of Eq. (20.b), is solved in two different ways. The transformed system for the fully developed velocity profile case has an analytical solution, which was presented in Eq. (29). For the case involving simultaneous development, the transformed system is solved through numerical routines for initial value problems. For the results of this work, the Gear method, implemented in the built-in function NDSolve of the software Mathematica v. 10.4 [43] was employed. After the transformed potentials $\bar{c}_{i}$ are obtained, the concentration is easily obtained through Eq. (20.b), with the summation truncated to an order $N$.

\subsection{Test cases}

At first, the influence of the Reynolds number upon the crossover phenomenon and the limiting current density scaling is investigated. Furthermore, a comparison of the results for the two flow development situations is performed in terms of both the concentration profiles and limiting current density.

The relative thickness of the porous electrode is also varied. Three different values for $w_{p}$ are used along the cases studied. The effects of the relative thickness on the region affected by the crossover at the centerline of the channel are investigated. Moreover, the variation of the limiting current density with $w_{p}$ is analyzed.

Table 1 summarizes the cases studied and the remainder of the required parameters, with typical values for membraneless RFBs, which are fixed throughout the simulated cases.

\section{Results and discussion}

\subsection{Eigenvalue problem}

Before proceeding with the analysis of the results for the transport of the species, the eigenfunctions must have the convergence assured. Amongst the parameters that vary along the test cases, only the relative thickness of the electrode, $w_{p}$, has influence over the eigenvalue problem of Eqs. (18.a-e). Tables 2a-2c show the evolution of the last five eigenvalues used in the eigenfunction expansion for the concentration in each case with the truncation order $M$. The reason why only the last five eigenvalues are presented is the observation, based on previously published results $[36,41]$, that their convergence is general slower than that of the earlier ones. All three values for the relative thickness specified on Table 1 are considered. Convergence to at least five significant digits is observed throughout Tables $2 \mathrm{a}-2 \mathrm{c}$.

Table 1

Definition of the fixed parameters and of the test cases (varying Re and $\mathrm{w}_{\mathrm{p}}$ ) for the analysis of membraneless RFBs in flow-by configuration.

\begin{tabular}{lllll}
\hline Parameter & Value & Test case & $R e$ & $w_{p}$ \\
\hline$\varepsilon$ & 0.8 & 1 & 5 & 0.5 \\
$D a$ & 0.002 & 2 & 10 & 0.5 \\
$x_{o}$ & 10 & 3 & 25 & 0.5 \\
$\sigma$ & 0.02 & 4 & 50 & 0.5 \\
$a h$ & 26.67 & 5 & 100 & 0.5 \\
$S c$ & 200 & 6 & 50 & 0.25 \\
& & 7 & 50 & 0.75 \\
\hline
\end{tabular}

\subsection{Effects of the Reynolds number}

Prior to the analysis of the results for the dimensionless concentration and related quantities, a verification of the concentrations convergence behavior must be carried out. Tables 3a-3c show the values of the bulk concentration for five selected longitudinal positions as a function of the truncation order $N$. Three Reynolds numbers are selected from the test cases of Table 1 . Only the convergence for the simultaneous development flow case is verified. The analytical solution of a decoupled transformed system for the fully developed flow case warrants faster convergence rates than for the simultaneous development case [30-33]. Convergence to at least three significant digits is observed for all three Reynolds numbers here analyzed.

Enough separation between the electrolytes and the electrode on the opposite side to the one they were injected is crucial to attain good operation with membraneless RFBs. Undesired contact of any electrolyte with the wrong electrode, known as crossover, can cause mixed potentials and severe loss of performance [1315]. Therefore, a careful analysis of the mixture zone at the interface between the two solutions at the centerline of the channel is carried out. Fig. 2a-c show the contour plots for the dimensionless concentration for three different Reynolds numbers $(R e=5,25$, 100 ) and a relative thickness of the electrode equal to 0.5 , assuming the simultaneous development flow condition. These graphs allow for a general overview of the concentration behavior over the whole domain. For a Reynolds number equal to 5, a significant amount of electrolyte is driven towards the electrode on the opposite side. This situation is not present for the other two Reynolds number studied, with the case of $R e=100$ showing a separation clearly larger amongst the cases depicted in Fig. 2.a-c. This behavior was expected, since smaller Reynolds number implicates higher residence times, which gives the transversal diffusion more time to occur. At the electrode adjacent to the electrolyte flow, Fig. 2.a-c show small protrusions near the entrance of the channel, increasing in size with higher Reynolds number. Such fact is an indication that the increase in the Reynolds number encourages a more significant portion of fluid rich in electrolytes to enter the porous electrode, with the protrusions being a direct result of transversal convective flux of species, included in the simultaneous development flow case. The remainder of the region of the electrode adjacent to the flow of electrolytes consumes the reactants that manage to penetrate into the porous medium almost entirely.

Fig. 3a-c show the mixture zone more clearly by presenting the dimensionless concentration profiles at the outlet of the channel for three selected Reynolds numbers and a relative electrode thickness of 0.5. Fig. 3.a corroborates the conclusion drawn from the contour plot of Fig. 2.a, showing that, for $R e=5$, a significant amount of electrolyte reaches the electrode on the opposite side, increasing the possibility of occurrence of mixed potentials. For $R e=100$, the separation is guaranteed, with almost null concentrations at the surroundings of the interface between free fluid and the electrode positioned at the opposite side to which the electrolytes were injected. The $R e=25$ case presents a somewhat intermediate behavior, with the moving front of the mixture zone approximately halfway between the centerline of the channel and the interface of the free fluid with the electrode. A comparison between the concentration profiles at the outlet of the channel for the simultaneous development (indicated as "SD" in Fig. $3 a-c$ ) and fully developed (indicated as "FD" in Fig. 3a-c) flow cases are also offered in Fig. 3a-c. The agreement between the two models for the velocity vector is perfect to the graph scale for this longitudinal position, indicating that the flow development is no longer important in evaluating the dimensionless concentration profile at the outlet of the channel. 
Table 2a

Convergence of the last five eigenvalues used in the eigenfunction expansion for the concentration in each case. $w_{p}=0.25$.

\begin{tabular}{|c|c|c|c|c|c|}
\hline & $\beta_{41}$ & $\beta_{42}$ & $\beta_{43}$ & $\beta_{44}$ & $\beta_{45}$ \\
\hline$M=100$ & 84.225 & 86.301 & 88.374 & 90.444 & 92.511 \\
\hline$M=105$ & 84.225 & 86.301 & 88.373 & 90.444 & 92.510 \\
\hline$M=110$ & 84.225 & 86.300 & 88.373 & 90.443 & 92.510 \\
\hline$M=115$ & 84.224 & 86.300 & 88.373 & 90.443 & 92.510 \\
\hline$M=120$ & 84.224 & 86.300 & 88.373 & 90.443 & 92.510 \\
\hline$M=125$ & 84.224 & 86.300 & 88.373 & 90.443 & 92.509 \\
\hline$M=130$ & 84.224 & 86.300 & 88.372 & 90.442 & 92.509 \\
\hline
\end{tabular}

Table 2b

Convergence of the last five eigenvalues used in the eigenfunction expansion for the concentration in each case. $w_{p}=0.5$.

\begin{tabular}{|c|c|c|c|c|c|}
\hline & $\beta_{51}$ & $\beta_{52}$ & $\beta_{53}$ & $\beta_{54}$ & $\beta_{55}$ \\
\hline$M=110$ & 146.714 & 149.701 & 153.820 & 157.445 & 163.149 \\
\hline$M=115$ & 146.175 & 149.045 & 151.936 & 155.367 & 158.637 \\
\hline$M=120$ & 146.161 & 148.902 & 151.695 & 154.539 & 157.619 \\
\hline$M=125$ & 146.157 & 148.895 & 151.673 & 154.496 & 157.348 \\
\hline$M=130$ & 146.156 & 148.895 & 151.673 & 154.490 & 157.338 \\
\hline$M=135$ & 146.155 & 148.894 & 151.672 & 154.489 & 157.337 \\
\hline$M=140$ & 146.154 & 148.893 & 151.672 & 154.489 & 157.337 \\
\hline
\end{tabular}

Table 2c

Convergence of the last five eigenvalues used in the eigenfunction expansion for the concentration in each case. $w_{p}=0.75$.

\begin{tabular}{|c|c|c|c|c|c|}
\hline & $\beta_{36}$ & $\beta_{37}$ & $\beta_{38}$ & $\beta_{39}$ & $\beta_{40}$ \\
\hline$M=125$ & 157.401 & 161.039 & 164.036 & 166.323 & 168.365 \\
\hline$M=130$ & 157.400 & 161.038 & 164.034 & 166.322 & 168.356 \\
\hline$M=135$ & 157.399 & 161.038 & 164.033 & 166.322 & 168.355 \\
\hline$M=140$ & 157.398 & 161.036 & 164.033 & 166.321 & 168.354 \\
\hline$M=145$ & 157.396 & 161.035 & 164.032 & 166.320 & 168.353 \\
\hline$M=150$ & 157.395 & 161.035 & 164.032 & 166.320 & 168.352 \\
\hline$M=155$ & 157.395 & 161.034 & 164.031 & 166.320 & 168.352 \\
\hline
\end{tabular}

Table 3a

Convergence of the eigenfunction expansion for the dimensionless bulk concentration for $R e=5$ and five different longitudinal positions.

\begin{tabular}{|c|c|c|c|c|c|}
\hline & $x=0.1$ & $x=0.2$ & $x=0.4$ & $x=0.6$ & $x=0.8$ \\
\hline$N=5$ & 0.449 & 0.454 & 0.457 & 0.454 & 0.451 \\
\hline$N=10$ & 0.474 & 0.471 & 0.466 & 0.460 & 0.455 \\
\hline$N=15$ & 0.481 & 0.474 & 0.467 & 0.460 & 0.455 \\
\hline$N=20$ & 0.483 & 0.476 & 0.467 & 0.460 & 0.455 \\
\hline$N=25$ & 0.483 & 0.476 & 0.467 & 0.460 & 0.455 \\
\hline
\end{tabular}

Table 3b

Convergence of the eigenfunction expansion for the dimensionless bulk concentration for $R e=25$ and five different longitudinal positions.

\begin{tabular}{|c|c|c|c|c|c|}
\hline & $x=0.1$ & $x=0.2$ & $x=0.4$ & $x=0.6$ & $x=0.8$ \\
\hline$N=20$ & 0.485 & 0.482 & 0.479 & 0.477 & 0.476 \\
\hline$N=25$ & 0.487 & 0.483 & 0.480 & 0.477 & 0.476 \\
\hline$N=30$ & 0.487 & 0.483 & 0.480 & 0.477 & 0.476 \\
\hline$N=35$ & 0.487 & 0.484 & 0.480 & 0.477 & 0.476 \\
\hline$N=40$ & 0.488 & 0.484 & 0.480 & 0.477 & 0.476 \\
\hline
\end{tabular}

Table 3c

Convergence of the eigenfunction expansion for the dimensionless bulk concentration for $R e=100$ and five different longitudinal positions.

\begin{tabular}{|c|c|c|c|c|c|}
\hline & $x=0.1$ & $x=0.2$ & $x=0.4$ & $x=0.6$ & $x=0.8$ \\
\hline$N=35$ & 0.489 & 0.485 & 0.482 & 0.481 & 0.481 \\
\hline$N=40$ & 0.489 & 0.486 & 0.483 & 0.482 & 0.481 \\
\hline$N=45$ & 0.489 & 0.487 & 0.483 & 0.482 & 0.481 \\
\hline$N=50$ & 0.489 & 0.488 & 0.484 & 0.483 & 0.481 \\
\hline$N=55$ & 0.489 & 0.488 & 0.485 & 0.483 & 0.481 \\
\hline
\end{tabular}



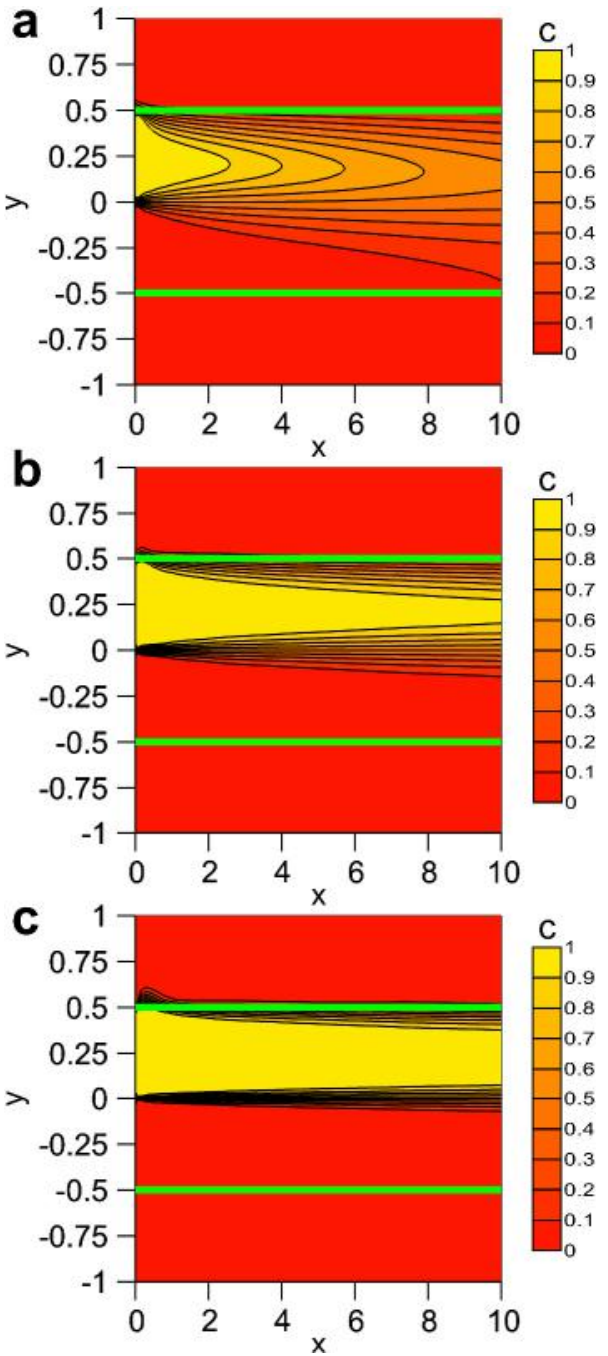

Fig. 2. Contour plots for the dimensionless concentration for three different Reynolds numbers and a relative thickness of the electrode equal to 0.5 . (a) $R e=5$; (b) $R e=25$; (c) $R e=100$. The simultaneous development flow condition was used in these results. Light green horizontal lines indicate the position of the interfaces between free fluid and the porous media.

Fig. 4a illustrates the variation of the dimensionless limiting current density with the electrode length for three different Reynolds numbers and relative thickness of the electrode equal to 0.5 under simultaneously developing flow conditions (filled symbols). Optimum lengths for the maximum current density are identified. At the region immediately after the entry, there is no significant amount of electrolytes within the electrode, and no current can be generated. As the length of the electrode is increased, the electrolytes reach the reactive surfaces of the electrode through both transversal diffusion and convection, and a current starts to form. This current has relatively high magnitudes, since the bulk concentration of electrolytes is still close to that initially provided and the difference from the concentration at the active sites of the electrode (zero in this work) is near the maximum possible value. For larger lengths, the depletion of electrolytes starts to gain prominence, decreasing the reaction rates and, consequently, the obtained current densities. Even though the total current generation continues to grow, the current density, being a measure of the average current generation along the domain, tends to decrease, which explains the behavior of all curves in Fig. 4a. Moreover, still in Fig. 4a, results obtained with the fully developed flow condition are included (unfilled symbols). As expected, little differ-
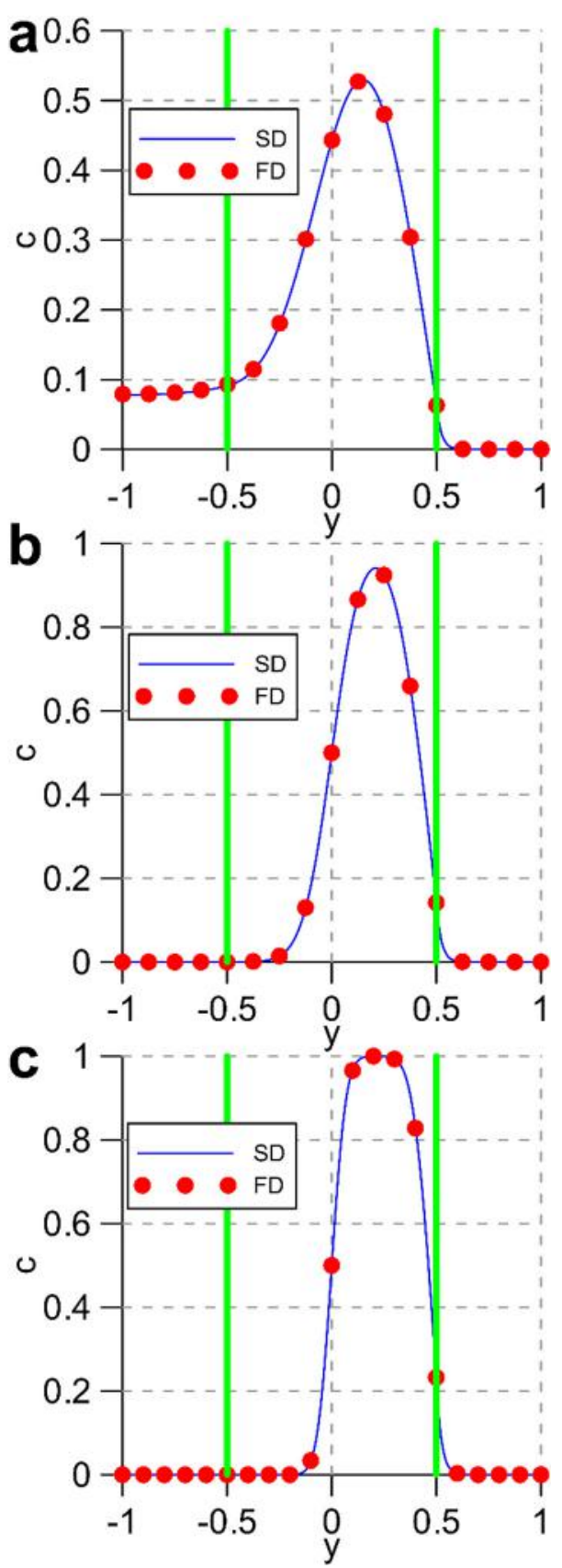

Fig. 3. Dimensionless concentration profiles at the outlet of the channel for three different Reynolds numbers and a relative thickness of the electrode equal to 0.5. (a) $R e=5$; (b) $R e=25$; (c) $R e=100$. Results for the fully developed flow case (named "FD") are included. Light green vertical lines indicate the position of the interfaces between free fluid and the porous media.

ence is noticed for longer electrodes, in comparison to the results for a simultaneous development flow condition. However, shorter electrodes implicate considerably larger deviations between the curves for the two flow conditions used in this work, including a significant shift on the optimum lengths for maximum limiting current density. The values for the limiting current density under simultaneous development flow condition are persistently higher for all electrode lengths studied, due to the presence of the transversal convective flux providing fresh reactants to the reaction sites, especially near the entry of the channel.

Fig. $4 \mathrm{~b}$ presents in a double-log plot a comparison of the scaling of the dimensionless limiting current density with the Reynolds 

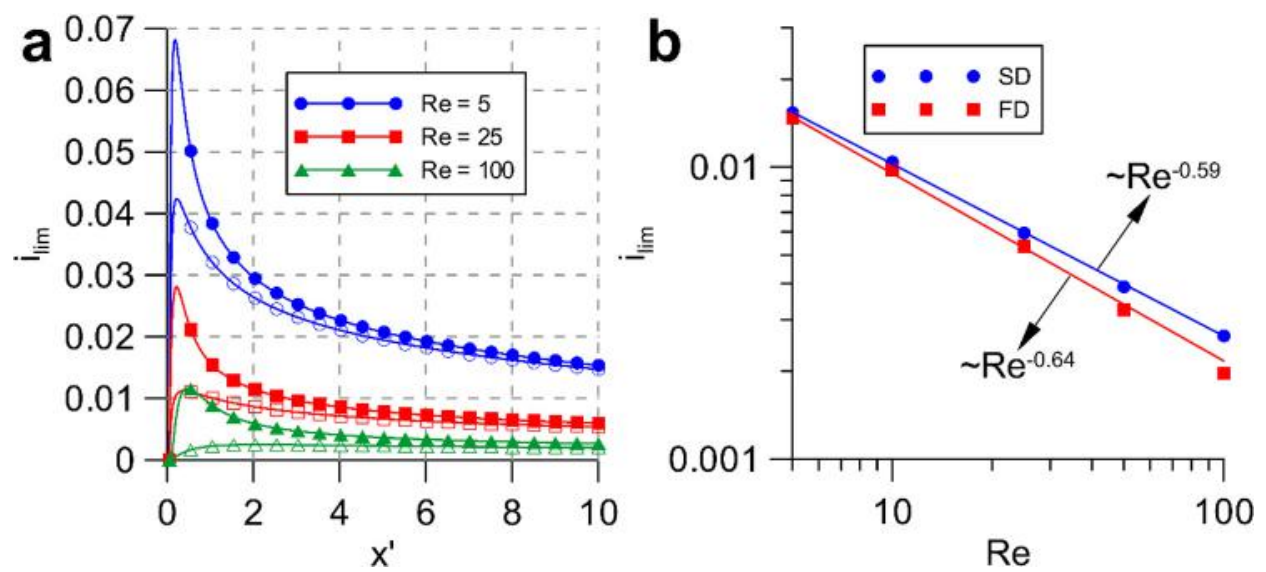

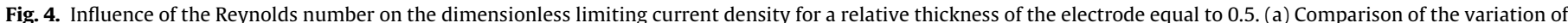

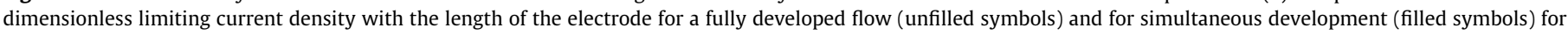

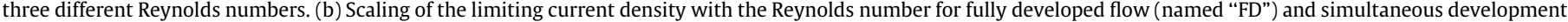
(named "SD").

Table 4a

Convergence of the dimensionless bulk concentration for $w_{p}=0.25$ and five different longitudinal positions.

\begin{tabular}{|c|c|c|c|c|c|}
\hline & $x=0.1$ & $x=0.2$ & $x=0.4$ & $x=0.6$ & $x=0.8$ \\
\hline$N=25$ & 0.488 & 0.488 & 0.488 & 0.489 & 0.489 \\
\hline$N=30$ & 0.490 & 0.489 & 0.489 & 0.489 & 0.489 \\
\hline$N=35$ & 0.491 & 0.489 & 0.489 & 0.489 & 0.488 \\
\hline$N=40$ & 0.491 & 0.490 & 0.489 & 0.489 & 0.488 \\
\hline$N=45$ & 0.492 & 0.490 & 0.489 & 0.489 & 0.488 \\
\hline
\end{tabular}

Table 4b

Convergence of the dimensionless bulk concentration for $w_{p}=0.5$ and five different longitudinal positions.

\begin{tabular}{|c|c|c|c|c|c|}
\hline & $x=0.1$ & $x=0.2$ & $x=0.4$ & $x=0.6$ & $x=0.8$ \\
\hline$N=35$ & 0.488 & 0.485 & 0.482 & 0.480 & 0.479 \\
\hline$N=40$ & 0.489 & 0.485 & 0.482 & 0.480 & 0.479 \\
\hline$N=45$ & 0.489 & 0.486 & 0.482 & 0.480 & 0.479 \\
\hline$N=50$ & 0.489 & 0.487 & 0.482 & 0.480 & 0.479 \\
\hline$N=55$ & 0.489 & 0.487 & 0.482 & 0.481 & 0.479 \\
\hline
\end{tabular}

Table 4c

Convergence of the dimensionless bulk concentration for $w_{p}=0.75$ and five different longitudinal positions.

\begin{tabular}{|c|c|c|c|c|c|}
\hline & $x=0.1$ & $x=0.2$ & $x=0.4$ & $x=0.6$ & $x=0.8$ \\
\hline$N=20$ & 0.476 & 0.465 & 0.446 & 0.434 & 0.426 \\
\hline$N=25$ & 0.477 & 0.470 & 0.452 & 0.439 & 0.429 \\
\hline$N=30$ & 0.477 & 0.472 & 0.454 & 0.440 & 0.430 \\
\hline$N=35$ & 0.477 & 0.472 & 0.455 & 0.440 & 0.430 \\
\hline$N=40$ & 0.477 & 0.472 & 0.455 & 0.441 & 0.430 \\
\hline
\end{tabular}

number between the cases with simultaneously developing flow (named "SD") and fully developed flow (named "FD") for a channel with dimensional length equal to 10 and relative thickness of the electrode equal to 0.5. Both curves are fitted to simulated data obtained for five different Reynolds numbers $(R e=5,10,25,50$, 100). The decrease in the dimensionless limiting current density with the Reynolds number is consistent with the previously noted interpretation of this quantity as a measure of the conversion of electrolytes along the channel. The higher the Reynolds number, less time the electrolytes spend inside the channel and, therefore, less time for the electrolytes to enter the electrode and suffer electrochemical reactions, decreasing the conversion. On the other hand, the limiting current density in its dimensional form is obtained from the dimensionless current density through the multiplication by a term directly proportional to the Reynolds number. Hence, the scaling shown in Fig. 4.b must be interpreted as a deviation, imposed by mass-transport-related deficiencies, of the dimensional limiting current density from the ideal linear scaling. The scaling of the dimensional limiting current density predicted then becomes $\sim R e^{0.41}$ and $\sim R e^{0.36}$ for the simultaneous development and fully developed flow conditions, respectively. The fact that the scaling is higher than $\sim R e^{0.33}$ typical of membraneless flow cells with flat electrodes [28], underscores the benefits of the use of porous electrodes for mass transport enhancement in these devices. 

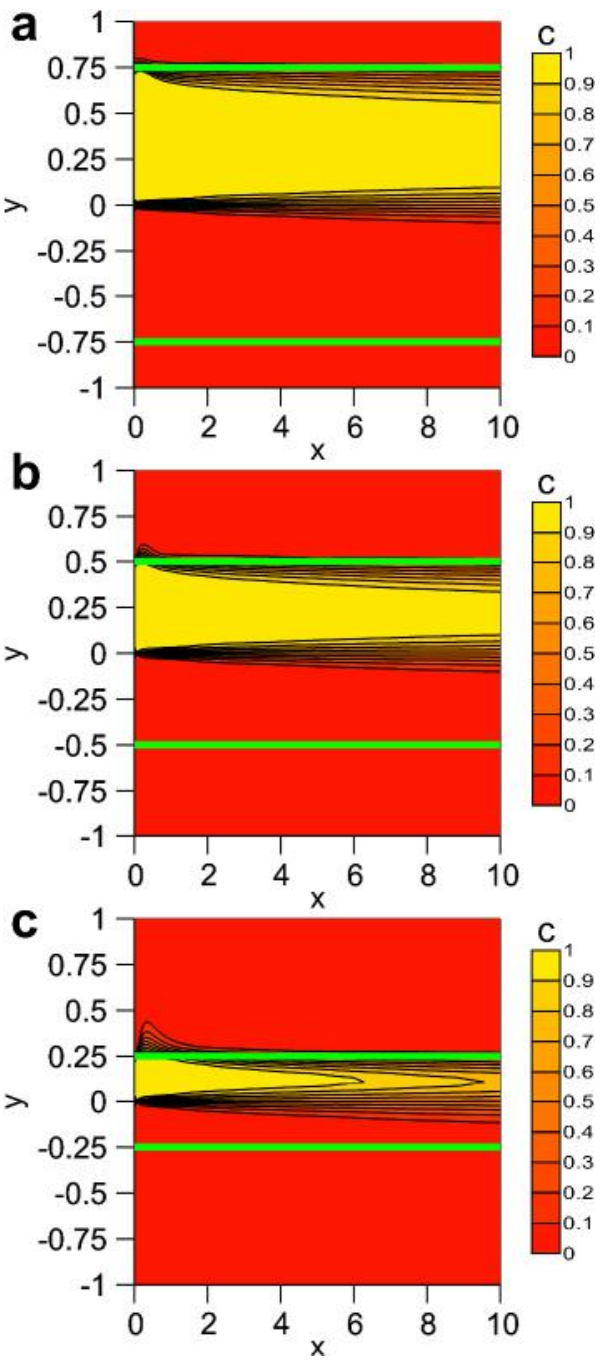

Fig. 5. Contour plots for the dimensionless concentration for three different relative thicknesses of the electrode and Reynolds equal to 50. (a) $w_{p}=0.25$; (b) $w_{p}=0.5$; (c) $w_{p}=0.75$. The simultaneous development flow condition was used in these results. Light green horizontal lines indicate the position of the interfaces between free fluid and the porous media.

\subsection{Effects of the relative thickness}

The convergence of the dimensionless concentration, this time for the cases varying the relative thickness of the electrode, are first evaluated. For a Reynolds number equal to 50 , three relative thicknesses are tested and the values for the concentration in selected longitudinal positions are obtained for ascending truncation order $N$, until certain criteria are satisfied. Tables $4 a-4 c$ summarize the results for the bulk concentration for the simultaneous development flow case, again considered to be the most critical situation. Convergence to three significant digits is attained for all three thicknesses for the truncation orders here indicated.

The need for an evaluation of the separation between the electrolyte and the electrode on the opposite side is the same as the one previously done with varying Reynolds number. Fig. 5a-c depict the contour plots of dimensionless concentration for three different relative thicknesses of the electrode defined in Table 1 and for a Reynolds number equal to 50 . For the case with electrodes occupying a quarter of the channel, the separation remains satisfactory for the conditions studied. The same seems to be the case for electrodes occupying half of the channel, with the contour corresponding to a dimensionless concentration equal to 0.1 suffi-
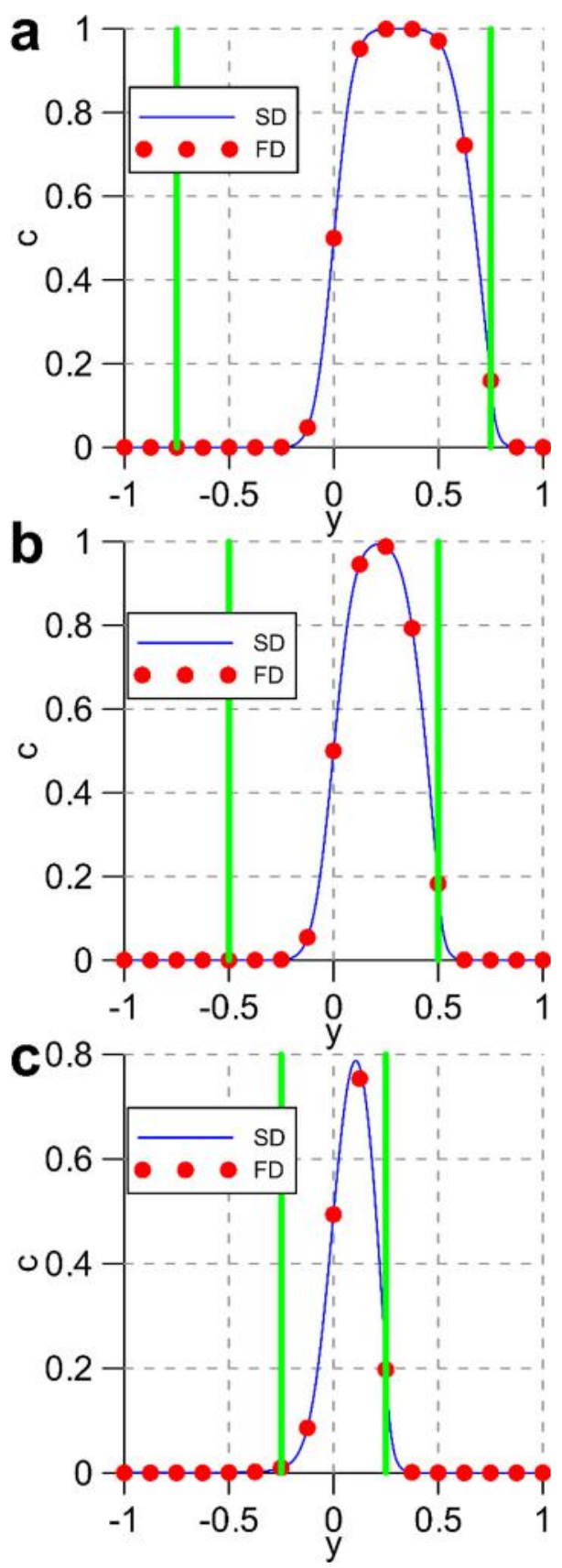

Fig. 6. Dimensionless concentration profiles at the outlet of the channel for three different relative thicknesses of the electrode and Reynolds number equal to 50. (a) $w_{p}=0.25$; (b) $w_{p}=0.5$; (c) $w_{p}=0.75$. Results for the fully developed flow case (named "FD") are included. Light green vertical lines indicate the position of the interfaces between free fluid and the porous media.

ciently apart from the interface between free fluid and the porous electrode on the opposite side of the one where the electrolytes are injected. Lastly, the case with electrodes occupying $75 \%$ of the channel has the smallest separation between the electrolyte and the electrode, with the contour equivalent to a dimensionless concentration of 0.1 almost touching the porous medium at the bottom. Such behavior is expected, since the increase of the proportion of the channel occupied by the porous electrodes reduces the space available for the free fluid. This fact decreases the transversal distance that the convection-diffusion mechanism must overcome in order to reach the electrode on the opposite side, facilitating the appearance of the mixed potentials phenomenon. 


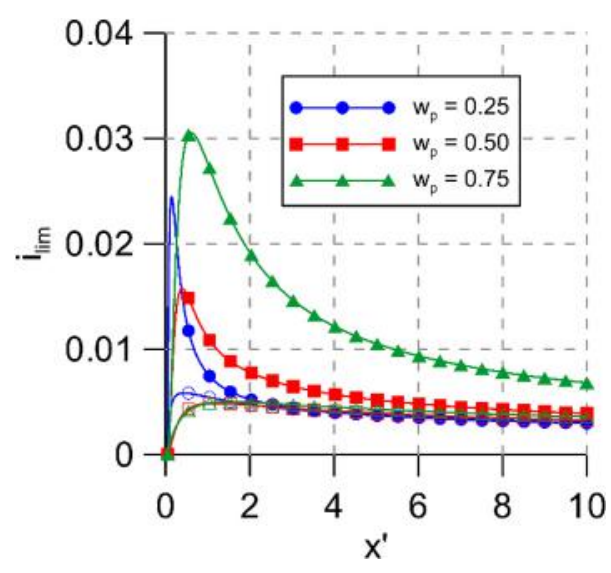

Fig. 7. Influence of the relative thickness of the electrode on the dimensionless limiting current density for a Reynolds number equal to 50. Comparison of the variation of dimensionless limiting current density with the length of the electrode for a fully developed flow (unfilled symbols) and for simultaneous development (filled symbols) for three different relative thicknesses.

Fig. $6 a-c$ show the influence of the relative thickness of the electrode on the mixture zone in more detail through concentration profiles at the outlet of the channel. The conclusions taken from Fig. 5.a-c remain valid. The mixture zone front with significant concentration of electrolytes is capable of reaching the porous electrode on the opposite side to the one where the reactants are injected, for the case with relative thickness of 0.75 . Such large thickness of the electrode becomes, for the conditions analyzed, inadequate for the prevention of the crossover and the associated phenomenon of mixed potentials. The other two thicknesses show a satisfactory separation of the electrolyte from the electrode at the bottom of Fig. 1, with the case in which the porous media occupy half of the channel showing an intermediate behavior.

Fig. 7 depicts the variation of the dimensionless limiting current density with the length of the electrode. Optimum lengths for maximum current densities are identified, being especially prominent for the results with the simultaneous development flow condition (filled symbols). The reasons for this phenomenon are the same already explored in Section 3.2. The results for the case with fully developed flow conditions (unfilled symbols) are almost insensitive to changes in the relative thickness of the electrodes for the situation studied. This behavior does not repeat itself for the simultaneous development results, highlighting the importance of considering the evolution of the velocity vector field along the channel. The simultaneous development flow conditions are particularly important for electrodes occupying $75 \%$ of the channel, case in which, even for longer electrodes, the difference between the current densities obtained with the two velocity vectors is very significant. Comparing the dimensionless limiting current density for different relative thickness of the porous media, becomes clear the increase in the conversion of the electrolyte with increasing thickness of the electrode. This allows for a conclusion that shorter, on the vicinity of the optimum length, and bulkier electrodes, though not thick enough to compromise the separation of the electrolytes, are beneficial from the point-of-view of mass transport only.

\section{Conclusions}

A theoretical analysis of the mass transport in a membraneless RFB in flow-by configuration employing porous electrodes in conditions of mass-transport-limitation was conducted. The methodology successfully employed in the solution of the governing equations was the Generalized Integral Transform Technique (GITT) with a single domain formulation strategy.
The analysis of the crossover dependency on the Reynolds number show that smaller values of Reynolds render inadequate separation between the electrolyte and the electrode on the opposite side, due to larger residence times inside the channel. This problem vanishes at the higher Reynolds number studied. As for the relative thickness of the electrode, an increase in its value had a negative effect on crossover prevention.

Optimum lengths for the electrode were identified for the maximum limiting current density in both analysis with varying Reynolds number and relative thickness of the electrode. The importance of the simultaneous flow development was demonstrated in a comparison with the current densities obtained under fully developed flow conditions.

The exponent of the scaling of the dimensionless limiting current density was identified as a deviation from the ideal linear scaling for the dimensional current density. Such deviation was due to mass-transport-related deficiencies, preventing the attainment of full conversion. The scaling obtained for the conditions studied was $\sim R e^{0.41}$, which contrasts with $\sim R e^{1 / 3}$ reported for flat electrodes [28], demonstrating the efficacy of the use of porous electrodes for the mitigation of mass transport limitations.

\section{Conflict of interest}

The authors declared that there is no conflict of interest.

\section{Acknowledgements}

The authors are grateful for the financial support offered by the Brazilian Government agencies CNPq, CAPES, and FAPERJ. KML is also grateful to Prof. Dimos Poulikakos and the Laboratory of Thermodynamics in Emerging Technologies at Eidgenössische Technische Hochschule Zürich for the hospitality during the time as visiting researcher as part of the PVE-CsF projects no. 401237/2014-1 and 207750/2015-7, sponsored by CNPq/Brazil.

\section{References}

[1] S. Chu, A. Majumdar, Opportunities and challenges for a sustainable energy future, Nature 488 (2012) 294-303, https://doi.org/10.1038/nature11475.

[2] D. Larcher, J.-M. Tarascon, Towards greener and more sustainable batteries for electrical energy storage, Nat. Chem. 7 (2015) 19-29, https://doi.org/10.1038/ nchem.2085.

[3] M. Skyllas-Kazacos, M. Rychcik, R.G. Robins, A.G. Fane, M.A. Green, New allvanadium redox flow cell, J. Electrochem. Soc. 133 (1986) 1057-1058, https:// doi.org/10.1149/1.2108706.

[4] A.Z. Weber, M.M. Mench, J.P. Meyers, P.N. Ross, J.T. Gostick, Q. Liu, Redox flow batteries: a review, J. Appl. Electrochem. 41 (2011) 1137-1164, https://doi.org/ 10.1007/s10800-011-0348-2.

[5] W. Wang, Q. Luo, B. Li, X. Wei, L. Li, Z. Yang, Recent progress in redox flow battery research and development, Adv. Funct. Mater. 23 (2013) 970-986, https://doi.org/10.1002/adfm.201200694.

[6] M.L. Perry, A.Z. Weber, Advanced redox-flow batteries: a perspective, J. Electrochem. Soc. 163 (2016) A5064-A5067, https://doi.org/10.1149/ 2.0101601jes.

[7] A. Stephan, B. Battke, M.D. Beuse, J.H. Clausdeinken, T.S. Schmidt, Limiting the public cost of stationary battery deployment by combining applications, Nat. Energy 1 (2016) 16079, https://doi.org/10.1038/nenergy.2016.79.

[8] C.S. Sharma, M.K. Tiwari, B. Michel, D. Poulikakos, Thermofluidics and energetics of a manifold microchannel heat sink for electronics with recovered hot water as working fluid, Int. J. Heat Mass Transf. 58 (2013) 135-151, https://doi.org/10.1016/j.ijheatmasstransfer.2012.11.012.

[9] J. Marschewski, R. Brechbühler, S. Jung, P. Ruch, B. Michel, D. Poulikakos, Significant heat transfer enhancement in microchannels with herringboneinspired microstructures, Int. J. Heat Mass Transf. 95 (2016) 755-764, https:// doi.org/10.1016/j.ijheatmasstransfer.2015.12.039.

[10] P. Ruch, T. Brunschwiler, W. Escher, S. Paredes, B. Michel, Toward fivedimensional scaling: how density improves efficiency in future computers, IBM J. Res. Dev. 55 (2011) 15:1-15:13, https://doi.org/10.1147/ JRD.2011.2165677.

[11] J. Marschewski, L. Brenner, N. Ebejer, P. Ruch, B. Michel, D. Poulikakos, 3Dprinted fluidic networks for high-power-density heat-managing miniaturized redox flow batteries, Energy Environ. Sci. 10 (2017) 780-787, https://doi.org/ 10.1039/C6EE03192G. 
[12] K.M. Lisboa, J. Marschewski, N. Ebejer, P. Ruch, R.M. Cotta, B. Michel, D. Poulikakos, Mass transport enhancement in redox flow batteries with corrugated fluidic networks, J. Power Sources 359 (2017) 322-331, https:// doi.org/10.1016/j.jpowsour.2017.05.038.

[13] R. Ferrigno, A.D. Stroock, T.D. Clark, M. Mayer, G.M. Whitesides, Membraneless vanadium redox fuel cell using laminar flow, J. Am. Chem. Soc. 124 (2002) 12930-12931, https://doi.org/10.1021/ja020812q.

[14] E.R. Choban, L.J. Markoski, A. Wieckowski, P.J.A. Kenis, Microfluidic fuel cell based on laminar flow, J. Power Sources 128 (2004) 54-60, https://doi.org/ 10.1016/j.jpowsour.2003.11.052.

[15] J.W. Lee, M.-A. Goulet, E. Kjeang, Microfluidic redox battery, Lab Chip 13 (2013) 2504, https://doi.org/10.1039/c3lc50499a.

[16] M. Zhang, M. Moore, J.S. Watson, T.A. Zawodzinski, R.M. Counce, Capital cost sensitivity analysis of an all-vanadium redox-flow battery, J. Electrochem. Soc. 159 (2012) A1183-A1188, https://doi.org/10.1149/2.041208jes.

[17] E. Kjeang, R. Michel, D.A. Harrington, N. Djilali, D. Sinton, A microfluidic fuel cell with flow-through porous electrodes, J. Am. Chem. Soc. 130 (2008) 40004006, https://doi.org/10.1021/ja078248c.

[18] O.A. Ibrahim, M.A. Goulet, E. Kjeang, In-situ characterization of symmetric dual-pass architecture of microfluidic co-laminar flow cells, Electrochim. Acta 187 (2016) 277-285, https://doi.org/10.1016/j.electacta.2015.11.081.

[19] M.A. Goulet, A. Habisch, E. Kjeang, In situ enhancement of flow-through porous electrodes with carbon nanotubes via flowing deposition, Electrochim. Acta 206 (2016) 36-44, https://doi.org/10.1016/j.electacta.2016.04.147.

[20] N. Da Mota, D.A. Finkelstein, J.D. Kirtland, C.A. Claudia, A.D. Stroock, H.D Abruña, Membraneless, room-temperature, direct borohydride/cerium fuel cell with power density of over $0.25 \mathrm{~W} / \mathrm{cm}^{2}$, J. Am. Chem. Soc. 134 (2012) 6076-6079, https://doi.org/10.1021/ja211751k.

[21] J. Marschewski, S. Jung, P. Ruch, N. Prasad, S. Mazzotti, B. Michel, D. Poulikakos, Mixing with herringbone-inspired microstructures: overcoming the diffusion limit in co-laminar microfluidic devices, Lab Chip 15 (2015) 1923-1933, https://doi.org/10.1039/C5LC00045A.

[22] J. Marschewski, P. Ruch, N. Ebejer, O.H. Kanan, G. Lhermitte, Q Cabrol, B. Michel, D. Poulikakos, On the mass transfer performance enhancement of membraneless redox flow cells with mixing promoters, Int. J. Heat Mass Transf. $106 \quad$ (2017) 884-894, https://doi.org/10.1016/j. ijheatmasstransfer.2016.10.030.

[23] M.E. Suss, K.M. Conforti, L. Gilson, C.R. Buie, M.Z. Bazant, Membraneless flow battery leveraging flow-through heterogeneous porous media for improved power density and reduced crossover, RSC Adv. 6 (2016) 100209-100213. https://doi.org/10.1039/c6ra22608f.

[24] A.A. Shah, M.J. Watt-Smith, F.C. Walsh, A dynamic performance model for redox-flow batteries involving soluble species, Electrochim. Acta 53 (2008) 8087-8100, https://doi.org/10.1016/j.electacta.2008.05.067.

[25] H. Al-Fetlawi, A.A. Shah, F.C. Walsh, Non-isothermal modelling of the allvanadium redox flow battery, Electrochim. Acta 55 (2009) 78-89, https://doi. org/10.1016/j.electacta.2009.08.009.

[26] A. Bazylak, D. Sinton, N. Djilali, Improved fuel utilization in microfluidic fuel cells: a computational study, J. Power Sources 143 (2005) 57-66, https://doi. org/10.1016/j.jpowsour.2004.11.029.
[27] D. Krishnamurthy, E.O. Johansson, J.W. Lee, E. Kjeang, Computational modeling of microfluidic fuel cells with flow-through porous electrodes, J. Power Sources 196 (2011) 10019-10031, https://doi.org/10.1016/j.jpowsour.2011.08.024.

[28] W.A. Braff, C.R. Buie, M.Z. Bazant, Boundary layer analysis of membraneless electrochemical cells, J. Electrochem. Soc. 160 (2013) A2056-A2063, https:/ doi.org/10.1149/2.052311jes.

[29] W.A. Braff, M.Z. Bazant, C.R. Buie, Membrane-less hydrogen bromine flow battery, Nat. Commun. 4 (2013) 1-6, https://doi.org/10.1038/ncomms3346.

[30] R.M. Cotta, Integral Transforms in Computational Heat and Fluid Flow, first ed. CRC Press, Boca Raton, FL, 1993.

[31] R.M. Cotta, Benchmark results in computational heat and fluid flow: the integral transform method, Int. J. Heat Mass Transf. 37 (1994) 381-393, https://doi.org/10.1016/0017-9310(94)90038-8.

[32] R.M. Cotta, M.D. Mikhailov, Heat Conduction: Lumped Analysis, Integral Transforms, Symbolic Computation, first ed., Wiley Interscience, Chichester, UK, 1997.

[33] R.M. Cotta, The Integral Transform Method in Thermal, Fluid Sciences and Engineering, first ed., Begell House, New York, NY, 1998.

[34] R.M. Cotta, D.C. Knupp, C.P. Naveira-Cotta, Analytical Heat and Fluid Flow in Microchannels and Microsystems, first ed., Springer, New York, NY, 2016.

[35] D.C. Knupp, C.P. Naveira-Cotta, R.M. Cotta, Theoretical analysis of conjugated heat transfer with a single domain formulation and integral transforms, Int Commun. Heat Mass Transf. 39 (2012) 355-362, https://doi.org/10.1016/j. icheatmasstransfer.2011.12.012.

[36] D.C. Knupp, C.P. Naveira-Cotta, R.M. Cotta, Conjugated convection-conduction analysis in microchannels with axial diffusion effects and a single domain formulation, J. Heat Transfer 135 (2013) 91401, https://doi.org/10.1115/ 1.4024425 .

[37] D.C. Knupp, R.M. Cotta, C.P. Naveira-Cotta, Heat transfer in microchannels with upstream-downstream regions coupling and wall conjugation effects, Numer. Heat Transf. Part B Fundam. 64 (2013) 365-387, https://doi.org/10.1080/ 10407790.2013.810535.

[38] D.C. Knupp, C.P. Naveira-Cotta, R.M. Cotta, Theoretical-experimental analysis of conjugated heat transfer in nanocomposite heat spreaders with multiple microchannels, Int. J. Heat Mass Transf. 74 (2014) 306-318, https://doi.org 10.1016/j.ijheatmasstransfer.2014.03.005.

[39] D.C. Knupp, R.M. Cotta, C.P. Naveira-Cotta, S. Kakaç, Transient conjugated heat transfer in microchannels: Integral transforms with single domain formulation, Int. J. Therm. Sci. 88 (2015) 248-257, https://doi.org/10.1016/j. ijthermalsci.2014.04.017.

[40] D.C. Knupp, R.M. Cotta, C.P. Naveira-Cotta, Fluid flow and conjugated heat transfer in arbitrarily shaped channels via single domain formulation and integral transforms, Int. J. Heat Mass Transf. 82 (2015) 479-489, https://doi. org/10.1016/j.ijheatmasstransfer.2014.11.007.

[41] K.M. Lisboa, R.M. Cotta, Hybrid integral transforms for flow development in ducts partially filled with porous media, Proc. Royal Soc. A. 474 (2018) 20170637, https://doi.org/10.1098/rspa.2017.0637.

[42] D.A.G. Bruggeman, Berechnung verschiedener physikalischer Konstanten von heterogenen Substanzen. I. Dielektriztätskonstanten und Leitfähigkeiten der Mischkörper aus isotropen Substanzen, Ann. Phys. 416 (1935) 636-664.

[43] S. Wolfram, Wolfram Mathematica, 2016, v. 10.4. 